\title{
Collaborative care at the crossroads ${ }^{\dagger}$
}

Peter Roy-Byrne

\section{Summary}

Collaborative care is a well-studied and effective model of integrating behavioural healthcare into primary care medical settings. Despite evidence of its effectiveness, it has been difficult to implement into the US healthcare system. The upcoming reorganisation of US healthcare will rely heavily on adaptations of this model to improve its uptake and costeffectiveness.

\section{Declaration of interest}

P.R.-B. is a consultant for Valant Medical Solutions.
Peter Roy-Byrne is Professor of Psychiatry at the University of Washington. His research focuses on ways to adapt evidence-based interventions for depression, anxiety and susbtance misuse to the primary care setting.

The collaborative care model for delivering evidence-based treatments for depression in the primary care setting, a model shown to be effective in multiple meta-analyses, is now almost 20 years old. ${ }^{1}$ This model was originally developed to address the problem that many patients with depression, unable to be seen by the inadequate number of mental health specialists in the USA, failed to receive effective treatment (principally medication) despite its wide availability. The collaborative care model emphasised the use of a care manager to facilitate measurementbased outcomes monitoring and patient self-management (e.g. treatment adherence), along with access to specialty consultation. Care manager and specialist also served to facilitate 'stepped care', where patients failing to improve were re-evaluated and then 'stepped up' to more or different treatments in an attempt to improve outcomes. In some later collaborative care model iterations, care managers delivered evidence-based psychotherapies, providing another treatment option. ${ }^{3}$ More recently, the model has been extended beyond depression to include patients with anxiety disorders, alcohol misuse, chronic pain and severe mental illness.

\section{Implementation challenges}

Despite the scientific evidence that this approach works, implementation of this model for mental disorders in the USA has been particularly difficult (it has been more easily implemented for chronic medical illnesses such as diabetes). The compromised motivational and cognitive states of patients, the existence of multiple psychiatric diagnoses demanding some kind of 'crossdiagnostic' or multiple comorbidity approach, the separation of behavioural and medical health systems and philosophies, and the fragmentation of the US mental health system, including the separation of substance misuse and mental health models and systems of care, have all contributed. A lack of organisational 'readiness to change' (including providers, ancillary staff, financing incentives and administrative processes) has also played a dominant role, along with concerns about cost.

A recent study of the uptake of three integrated mental healthcare models in one of the few US single organisation, single-payer health systems, the Veterans Health Administration, ${ }^{4}$ showed that adoption was greatest (47\%) for a crude 'collocation' model (mental health specialist in the clinic) compared with two models

†See pp. 132-139, this issue. requiring more change in the care process (17\% and $7 \%)$. These low-uptake models were more similar to the collaborative care model and likely to be more effective, and yet were much harder to implement. Cost concerns will be even more paramount with the upcoming reorganisation of US healthcare, posing a risk that watered-down versions of the collaborative care model that have not been scientifically tested will be adopted for expediency. Measurement-based outcome tracking will certainly survive because, with electronic medical record improvements, it will be cost-neutral. In contrast, the type and extent of care manager training and the nature and extent of expert consultation (both carrying significant costs) will be debated. Although a recent meta-analysis of the collaborative care model calls for more research to test 'moderators and mechanisms,' resources and time for more research and, given the adaptations required for implementation, it is certain that adoption will proceed as it usually does in business, with iterative qualitative improvement efforts to test what works and what is cost-effective in that business context. The rise of accountable care organisations will hopefully link payment to outcomes and drive further use of this model. Consistent with this, a study ${ }^{5}$ nicely demonstrated that pay for performance can improve patient follow-up and depression outcomes using this model.

\section{Collaborative care facilitation}

The study by Oosterbaan and colleagues, ${ }^{6}$ in this issue of the Journal, contains some novel and important elements that should be carefully considered as healthcare organisations look for better ways to implement the collaborative care model. These include its cluster-randomised design (by clinic rather than patient), its broad-based diagnostic focus, its formal inclusion of and linkage with the specialty mental healthcare system, its inclusion of a formal symptom severity strategy for assigning the initial 'step' of care, and its use of less-costly guided self-help workbooks to facilitate entry-level treatment. If there are to be any further randomised controlled trials of the collaborative care model, they should only occur in large systems that can randomise by clinic rather than patient, as Oosterbaan et al did, making the study of possible moderators of uptake possible. Identifying organisational aspects that facilitate adoption will likely be more crucial than studies of patient-level moderators. Although Oosterbaan et al broaden the focus of the collaborative care model beyond depression to anxiety and stress, further broadening is needed to include both common behavioural (e.g. substance misuse, pain) and medical (e.g. diabetes, cardiac disease) comorbidities (see the work now being done in implementing the Katon TEAMcare model of collaborative care for patients with depression, diabetes 
and heart disease $\left.{ }^{7}\right)$. The collaborative care model must link strongly with available specialty care (likely more easy in the European setting than in the Balkanised US system), and must exploit the use of technology in novel ways. The workbook strategy employed here could be done via web-based application. A recent study ${ }^{8}$ has suggested that centralised care manager and specialist availability (these could be done via web or videoconferencing platforms), could be more efficient, cheaper and even more effective in rural areas, removing the need for each clinic and system to incorporate new personnel. In contrast to the method used by Oosterbaan et al, one study suggests that severity of symptoms may not be an ideal method for determining who would best respond to a first step of care - i.e. patients who are more severely symptomatic may also respond to initial step care. ${ }^{9}$ Finally, although the short-term results of Oosterbaan et al's study are encouraging, the absence of longer-term benefit is concerning. This calls to mind the literature on surgery for low back pain, which shows improved outcomes in the short run but not at 1 year. It will be hard to recommend a model of service delivery without significant staying power, even as we understand these results as the outcome of gradual improvement in usual care patients over time. However, other collaborative care model studies have shown continued beneficial effects at 1 year, albeit with lower effect sizes, ${ }^{3,10}$ so the jury is still out.

In the USA, we now await a grand experiment where we try to provide physical and mental healthcare for larger numbers of citizens, and to find ways to more efficiently and cost-effectively provide the best possible care. It seems a certainty that the collaborative care model will be employed in various forms and iterations to facilitate initial behavioural healthcare access, to promote astute use of evidence-based treatment in primary care, and to identify and treat patients in need of further stepped care in primary care or specialty settings. There will be challenges in determining whether the collaborative care model is a 'one size fits all' solution, i.e. whether it will provide benefit with certain types of patient populations or care delivery systems (see a recent failure with patients with post-traumatic stress disorder ${ }^{11}$ ), and if and how much it can supplant or replace mental health specialty care. To ease implementation, future iterations might consider centralised care management using expert care managers and psychiatrists who communicate via telephone, text messaging, email or audio and/or video conferencing over the internet. This would not require a change in clinic personnel or functions, would allow delivery of both cognitive-behavioural therapy and expert medication management, and might simulate more 'specialty'oriented care, while at the same time achieving integration with primary medical care.

Peter Roy-Byrne, MD, University of Washington School of Medicine, At Harborview Medical Center, Box 35-9911, 325 9th Avenue, Seattle, Washington, USA. Email: roybyrne@u.washington.edu

First received 31 Mar 2013, accepted 25 Apr 2013

\section{References}

1 Katon W, von Korff M, Lin EH, Walker ED, Simon GE, Bush TM, et al. Collaborative management to achieve treatment guidelines. Impact on depression in primary care. JAMA 1995; 273: 1026-31.

2 Woltmann E, Grogan-Kaylor A, Perron B, Georges H, Kilbourne AM, Bauer MS. Comparative effectiveness of collaborative chronic care models for mental health conditions across primary, specialty, and behavioral health care settings: systematic review and meta-analysis. Am J Psychiatry 2012; 169 790-804.

3 Roy-Byrne P, Craske MG, Sullivan G, Rose RD, Edlund MJ, Lang AJ, et al. Delivery of evidence-based treatment for multiple anxiety disorders in primary care: a randomized controlled trial. JAMA 2010; 303: 1921-8.

4 Chang ET, Rose DE, Yano EM, Wells KB, Metzger ME, Post EP, et al. Determinants of readiness for primary care-mental health integration (PC-MHI) in the VA Health Care System. J Gen Intern Med 2013; 28: 353-62.

5 Unutzer J, Chan YF, Hafer E, Knaster J, Shields A, Powers D, et al. Quality improvement with pay-for-performance incentives in integrated behavioral health care. Am J Public Health 2012; 102: e41-5.

6 Oosterbaan D, Verbraak MJPM, Terluin B, Hoogendoorn AW, Peyrot WJ, Muntingh $A$, et al. Collaborative stepped care $v$. care as usual for common mental disorders: 8-month, cluster randomised controlled trial. $\mathrm{Br} J$ Psychiatry 2013; 203: 132-9.

7 Katon WJ, Lin EH, Von Korff M, Ciechanowski P, Ludman EJ, Young B, et al. Collaborative care for patients with depression and chronic illnesses. $N$ Engl J Med 2010; 363: 2611-20.

8 Fortney JC, Pyne JM, Mouden SB, Mittal D, Hudson TJ, Schroeder GW, et al. Practice-based versus telemedicine-based collaborative care for depression in rural federally qualified health centers: a pragmatic randomized comparative effectiveness trial. Am J Psychiatry 2013; 170: 414-25.

9 Bower P, Kontopantelis E, Sutton A, Kendrick T, Richards DA, Gilbody S, et al. Influence of initial severity of depression on effectiveness of low intensity interventions: meta-analysis of individual patient data. BMJ 2013; 346: f540.

10 Unützer J, Katon W, Callahan CM, Williams JWJ, Hunkeler E, Harpole L, et al. Collaborative care management of late-life depression in the primary care setting. JAMA 2002; 288: 2836-45.

11 Schnurr PP, Friedman MJ, Oxman TE, Dietrich AJ, Smith MW, Shiner B, et al. RESPECT-PTSD: re-engineering systems for the primary care treatment of PTSD, a randomized controlled trial. J Gen Intern Med 2013; 28: 32-40. 